Cite this: RSC Adv., 2014, 4, 11121

\title{
Light absorption of poly(3-hexylthiophene) single crystals $\uparrow$
}

Received 26th November 2013

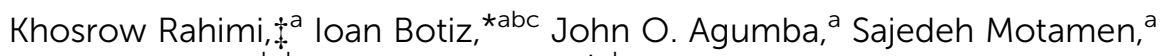
Natalie Stingelin ${ }^{\text {bd }}$ and Günter Reiter ${ }^{\star a b}$

Accepted 10th February 2014

DOI: $10.1039 / c 3 r a 47064 d$

www.rsc.org/advances

We report the local UV-Vis absorption behaviour of single crystals of conjugated poly-3-hexylthiophene (P3HT), obtained by crystallization in dilute solutions at elevated temperatures based on a self-seeding approach and characterized by high internal structural order.

Conjugated polymers represent promising active systems for a variety of optoelectronic applications, such as solar cells, ${ }^{1}$ light emitting diodes, ${ }^{2}$ field-effect transistors, ${ }^{3-5}$ etc. However, the performance of these materials in such devices has so far often remained poor, mostly due to the difficulties in controlling molecular conformations, structural packing, and morphology $y^{6,7}$ of these polymers. In order to correctly interpret and finally to improve the functional behavior of conjugated polymers, it is thus necessary to unravel the relationship between the light absorption properties and molecular conformation or polymer microstructure. As a suitable model system for a systematic study of such structure-optoelectronic property relations, we have recently identified highly ordered single crystals of conjugated poly-3-hexylthiophene (P3HT) of weight average molecular weight of $26400 \mathrm{~g} \mathrm{~mol}^{-1}$, characterized by a unique molecular conformation on all length-scales. ${ }^{8}$ They were obtained via crystallization in solution by a self-seeding technique. These crystals are composed of closely packed, $\pi-\pi$ stacked ( $\pi-\pi$ distance of $\sim 0.33 \mathrm{~nm}$ ) fully extended chains which are oriented perpendicular to the substrate. ${ }^{8}$

${ }^{a}$ Institute of Physics, University of Freiburg, Hermann-Herder Strasse 3, Freiburg 79104, Germany. E-mail: ioan.botiz@scientist.com; guenter.reiter@physik. uni-freiburg.de

${ }^{b}$ Freiburg Institute for Advanced Studies, Albertstrasse 19, Freiburg 79104, Germany ${ }^{c}$ Babes-Bolyai University, Faculty of Physics and Interdisciplinary Research Institute in Bio-Nano-Sciences, Treboniu Laurian Str. 42, Cluj Napoca 400271, Romania

${ }^{d}$ Department of Materials, Imperial College London, Exhibition Road, London SW7 $2 A Z, U K$

$\dagger$ Electronic supplementary information (ESI) available: Information about polymer materials and experimental methods as well as detailed information on crystal washing procedure are provided. See DOI: 10.1039/c3ra47064d

\$ Present Address: DWI-Leibniz Institute for Interactive Materials, Forckenbeckstr. 50, 52056 Aachen, Germany.
Fig. 1 compares the UV-Vis absorbance spectrum recorded from a small area on an as-prepared, unwashed single crystal of P3HT deposited on a glass substrate with the ones obtained from a homogeneous solution of P3HT (in 3-hexyl-thiophene) and a thin, spin-cast film on glass. While the solution spectrum only exhibits a single broad absorption peak at short wavelength $(455 \mathrm{~nm})$, mainly related to intra-chain states of individual P3HT chains in a flexible random-coil conformation, ${ }^{9}$ the spin-cast thin film shows the characteristic transitions related to inter-chain exciton delocalization and a significant coupling to vibrations. The ratio of the amplitudes of the $\mathrm{A}_{0-0}(603 \mathrm{~nm})$ and the $\mathrm{A}_{0-1}(555 \mathrm{~nm})$ peaks which is smaller than $1(\sim 0.80 \pm$ $0.05)$ is characteristic for partially ordered H-aggregate-like chains coexisting with non-aggregated chain sequences. ${ }^{10,11}$

In strong contrast, the absorption spectrum of the unwashed P3HT single crystal reveals an $\mathrm{A}_{0-0} / \mathrm{A}_{0-1}$ intensity ratio larger than $1(\sim 2.20 \pm 0.15)$. This large intensity ratio might be a consequence of long-range intra-chain interactions along P3HT

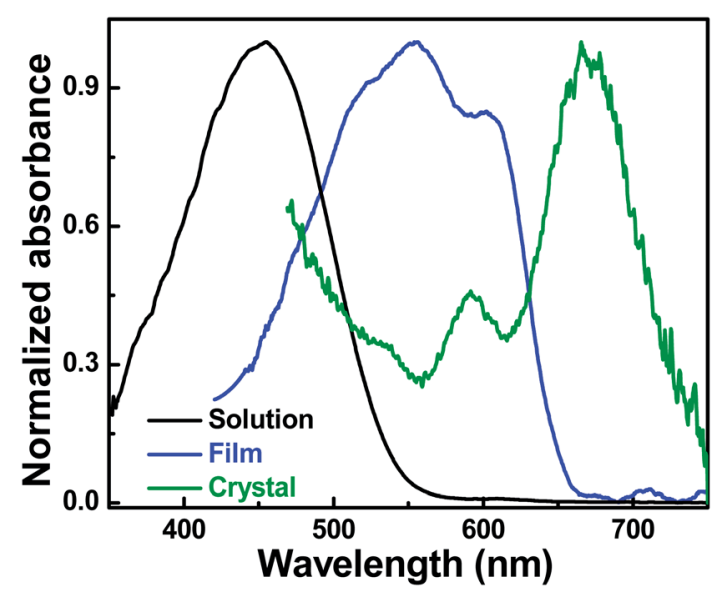

Fig. 1 Normalized UV-Vis absorption spectra of P3HT: 3-hexylthiophene solution $\left(0.2 \mathrm{mg} \mathrm{ml}^{-1}\right)$ at $50{ }^{\circ} \mathrm{C}$ (black), thin film of a thickness of $\sim 65 \mathrm{~nm}$ spin-cast from a homogeneous solution in 3-hexylthiophene (blue) and a single crystal of a thickness of $\sim 59 \mathrm{~nm}$ (olive) spin cast from a 3-hexylthiophene dispersion. 
chains that are closely packed, fully extended/planarized and orthogonally oriented with respect to the substrate. ${ }^{8}$ This result is in accordance with previously reported absorption spectra of defect free highly regioregular P3HT films that also showed a dominant 0-0 transition. ${ }^{12}$

Interestingly, with respect to spin-cast thin films the P3HT single crystal showed a remarkable red shift of $\sim 70 \mathrm{~nm}$ for the 0-0 transition (Fig. 1). P3HT aggregates can consist of crystalline regions, amorphous domains or a combination of both. Amorphous chain sequences lead to structural defects, grain boundaries and coiled-like chain conformations that can reduce intra-chain order and therefore, UV-Vis absorption signatures are broadened and blue shifted towards higher energies. ${ }^{13}$ This is not the case for single crystals of P3HT. As single crystals are predominantly free of grain boundaries or structural defects and essentially do not contain amorphous regions, features of the absorption spectrum can be clearly correlated to structural aspects. Thus, the red shift observed in Fig. 1 may be related to the fully extended chains of P3HT single crystals $^{8}$ that are highly planarized and thus, can allow for fully delocalized states along the backbone of P3HT. ${ }^{14}$ It is important to note that the magnitude of the red shift observed in single P3HT crystals compared to thin as cast films is surprising. Red shift is often observed in as cast thin films of P3HT when varying molecular conformations and aggregation properties, ${ }^{15}$ but to date the magnitude of red shifts observed for P3HT structures was much less than $70 \mathrm{~nm}$, even when dealing with highly ordered P3HT fibers. ${ }^{16,17}$

The vibronic peaks ( $A_{0-0}$ and $A_{0-1}$ in olive spectrum in Fig. 1) are separated by $\sim 78 \mathrm{~nm}$ which corresponds to an energy difference of $\sim 240 \mathrm{meV}$. This value is higher than the value of one vibrational quantum ( $\sim 170$ to $180 \mathrm{meV}$ ) that was reported in literature for thiophene based aggregates. ${ }^{18-20}$ Such a high value may suggest that in these form-II P3HT single crystals with interdigitated side chains, the packing may lead to backbones slightly shifted with respect to each other both along the directions of the molecular long and short axes. Such particular molecular packing cannot exclude closer distances between molecules and thus possibly some inter-chain orbital overlap (an electron in a single P3HT crystal could now be characterized by a double delocalization along the chains and in between the chains).

In order to probe the relationship between the polymer chain conformations and absorption properties, we have compared local UV-Vis absorption spectra (exposure time: $300 \mathrm{~ms}$ ) from as-deposited P3HT single crystals surrounded by additional polymers with the ones from washed crystals. In addition, optical micrographs using crossed polarizers were taken which demonstrated that after efficient washing the birefringence over the whole crystal showed a significantly higher and uniform brightness. Birefringence of as-deposited crystals exhibited much lower brightness and contained spots of poor contrast which we attribute to patches of chains deposited on the crystal surface (see Fig. S1 in the ESI $\dagger$ ).

Fig. 2 shows the differences in absorption of an as-deposited crystal (olive curve) surrounded by less ordered polymer fraction and a washed single crystal (red curve). In comparison to the

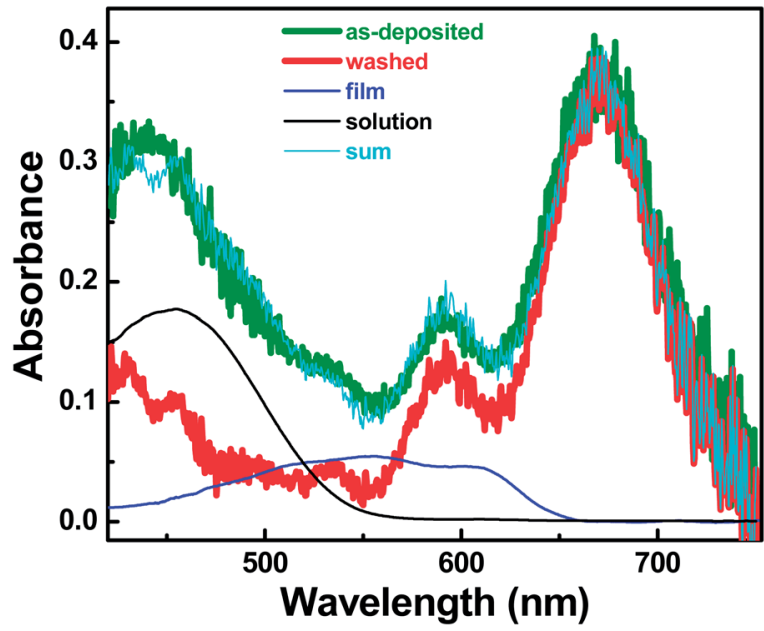

Fig. 2 Absorption spectrum of an as-deposited P3HT crystal surrounded by additional less ordered polymer fraction (olive), compared with the spectrum of a washed P3HT single crystal (red). We note that additional molecules surrounding the P3HT single crystal were washed away before recording the absorption spectrum (details on crystal washing are given in the ESI $\uparrow$ ). Black and blue curves represent spectra of $\mathrm{P} 3 \mathrm{HT}$ in solution and a thin film as shown in Fig. 1. In order to represent the difference between the washed and as deposited single crystal, these curves have been weighted by $0.12 \pm 0.02$ and $0.11 \pm$ 0.02 , respectively. The cyan line represents the result of the sum of appropriately weighted curves corresponding to a washed single crystal, a thin film and a solution, respectively.

washed crystal, the as-deposited crystal exhibits significant absorption at high energies. Moreover, the reduced $\mathrm{A}_{0-0} / \mathrm{A}_{0-1}$ ratio suggests a decrease of intra-chain exciton delocalization.

It is important to note that washing of an as-deposited crystal did not lead to a shift of the peak positions, suggesting that the measured spectrum may be a superposition of different contributions, which could be partially removed by the washing. In order to identify these contributions, we have tested if the spectrum of the as-deposited crystal could be reconstructed on the basis of the spectra presented in Fig. 1. To this end, we have weighted these spectra, reflecting the amount of absorbing P3HT molecules. The closest overlap with the spectrum of the as-deposited crystal was obtained by the sum of the three spectra of Fig. 1, where the spectrum for the P3HT solution was weighted by $0.12 \pm 0.02$, the spectrum for the thin film by $0.11 \pm 0.02$ and the one for the washed single crystal by 1 . The resulting composite curve (cyan) is almost identical to the one of the as-deposited single crystal (olive). We therefore conclude that the as-deposited single crystal was surrounded by fractions of $11 \%$ and $12 \%$ of $\mathrm{P} 3 \mathrm{HT}$ molecules that adopt conformations like in films and solution, respectively. These fractions correspond roughly to an average thickness of about $6 \mathrm{~nm}$. These values are consistent with a layer of about $10 \mathrm{~nm}$ of amorphous or aggregated polymers on top of P3HT single crystals before washing as observed by atomic force microscopy (AFM) studies (see Fig. S2 in the ESI†). These studies thus show that a layer of about 8-12 $\mathrm{nm}$ in thickness was removed from the top of $\sim 59 \mathrm{~nm}$ thick single crystals in the course of the washing procedure. 
While a $6 \mathrm{~nm}$ thick layer comprised of aggregated P3HT chains may be expected on top of the crystal as a result of the deposition process of single crystal from the dispersion, the additional $6 \mathrm{~nm}$ thick layer of polymer chains exhibiting a spectrum similar to well dissolved molecules in solution (i.e. isolated, coiled P3HT chains) is surprising. It is unclear how solution-like conformations can be preserved after deposition and subsequent solvent evaporation. Annealing experiments on single crystals before washing are currently performed to shed light on this issue.

\section{Conclusions}

We have demonstrated that perfect ordering and stretching of P3HT chains in a single crystal is leading to a significantly different light absorption behavior. The washing experiments showed that absorption properties of as deposited dispersion of P3HT single crystals can be attributed to three different contributions, related to different morphologies. Washed P3HT single crystals are absorbing light at higher wavelengths compared to spin cast films and thus a remarkable red shift of $\sim 70 \mathrm{~nm}$ is observed. In addition, in comparison to spin cast films deposited from a homogeneous solution or for crystalline fibers, ${ }^{15,16}$ the $\mathrm{A}_{0-0} / \mathrm{A}_{0-1}$ intensity ratio for single crystals is significantly larger. This suggests the presence of strong intrachain interactions along the (fully) planarized chains, allowing for large conjugation lengths and possibly delocalized states along the whole chain. The large energy separation of the vibronic peaks with respect to the reported vibrational spectra might be related to the type of packing induced by interdigitated hexyl-side-groups of the studied form-II single crystals of P3HT which may allow for some inter-chain orbital overlap.

\section{Notes and references}

1 G. Yu, J. Gao, J. C. Hummelen, F. Wudl and A. J. Heeger, Science, 1995, 270, 1789.

2 J. H. Burroughes, D. D. C. Bradley, A. R. Brown, R. N. Marks, K. Mackay, R. H. Friend, P. L. Burns and A. B. Holmes, Nature, 1990, 347, 539.
3 F. Padinger, R. S. Rittberger and N. S. Sariciftci, Adv. Funct. Mater., 2003, 13, 85.

4 C. Tanase, E. J. Meijer, P. W. M. Blom and D. M. d. Leeuw, Phys. Rev. Lett., 2003, 91, 216601.

5 R. J. Kline, M. D. McGehee, E. N. Kadnikova, J. Liu and J. M. J. Fréchet, Adv. Mater., 2003, 15, 1519.

6 F. C. Grozema and L. D. A. Siebbeles, J. Phys. Chem. Lett., 2011, 2, 2951.

7 I. Botiz and S. B. Darling, Macromolecules, 2009, 42, 8211.

8 K. Rahimi, I. Botiz, N. Stingelin, N. Kayunkid, M. Sommer, F. P. V. Koch, H. Nguyen, O. Coulembier, P. Dubois, M. Brinkmann and G. Reiter, Angew. Chem., Int. Ed., 2012, 51, 11131.

9 G. W. Heffner and D. S. Pearson, Macromolecules, 1991, 24, 6295.

10 M. Baghgar, J. Labastide, F. Bokel, I. Dujovne, A. McKenna, A. M. Barnes, E. Pentzer, T. Emrick, R. Hayward and M. D. Barnes, J. Phys. Chem. Lett., 2012, 3, 1674.

11 F. C. Spano, Acc. Chem. Res., 2009, 43, 429.

12 P. Kohn, S. Huettner, H. Komber, V. Senkovskyy, R. Tkachov, A. Kiriy, R. H. Friend, U. Steiner, W. T. S. Huck, J. Sommer and M. Sommer, J. Am. Chem. Soc., 2012, 134, 4790.

13 J. Clark, C. Silva, R. H. Friend and F. C. Spano, Phys. Rev. Lett., 2007, 98, 206406.

14 C. Hellmann, F. Paquin, N. D. Treat, A. Bruno, L. X. Reynolds, S. A. Haque, P. N. Stavrinou, C. Silva and N. Stingelin, Adv. Mater., 2013, 25, 4906.

15 J. Clark, J.-F. Chang, F. C. Spano, R. H. Friend and C. Silva, Appl. Phys. Lett., 2009, 94, 163306.

16 E. T. Niles, J. D. Roehling, H. Yamagata, A. J. Wise, F. C. Spano, A. J. Moule and J. K. Grey, J. Phys. Chem. Lett., 2012, 3, 259.

17 J. D. Roehling, I. Arslan and A. J. Moule, J. Mater. Chem., 2012, 22, 2498.

18 F. C. Spano, Acc. Chem. Res., 2010, 43, 429.

19 F. C. Spano, Annu. Rev. Phys. Chem., 2006, 57, 217.

20 M. D. Barnes and M. Baghar, J. Polym. Sci., Part B: Polym. Phys., 2012, 50, 1121. 